\title{
Mirror feedback assisted recovery from hemiparesis following stroke. In Reply to Morkisch et al.: How to perform mirror therapy after stroke? Evidence from a meta-analysis
}

\author{
Vilayanur Subramanian Ramachandran* and Diane Rogers-Ramachandran \\ Salk Institute for Biological Sciences, La Jolla, CA, United States
}

\begin{abstract}
Of the 2/3rd who survive, half will be permanently disabled (World Stroke Organization). Given these alarming statistics, it's not surprising that tremendous of amounts of time, resources and funding have been devoted towards research into stroke rehab. In view of this, simple, easy-to-implement procedures are highly sought after. One such procedure is the use of visual feedback conveyed by a mirror, which seems to reactivate some dormant pathways in patients who have suffered a recent stroke (Altschuler et al., 1999; Ramachandran \& Altschuler, 2009). This procedure has been validated in several dozen clinical trials, of which a substantial number were conducted rigorously. Morkisch et al. (2019) now present a systematic review and meta-analysis of this dense literature pertaining to this topic. They conclude that "there is a high level evidence for mirror therapy's effectiveness in treating post-stroke hemiparesis". A novel conclusion that emerged from this meta-analysis was that the larger the mirror the more effective the treatment is. Additionally, if an object is manipulated by the normal hand and its reflection viewed in the mirror, the procedure is not as effective, possibly because the discrepancy of signals between the visual feedback (reflection of the object) and the lack of confirmatory somatosensory input from the affected limb leads to inhibition rather than synergy. Lastly, for reasons yet unclear, sending movement commands to the unaffected hand alone (unilateral) is more effective than sending bilateral commands, contrary to the original protocol.

Taken collectively, research in this field has two implications - First, the immediate practical utility in the clinic by optimizing mirror therapy's efficacy for hemiparesis after stroke. Second, it leads to a rejection of the model of the brain as made up of isolated, autonomous modules, towards a more dynamic picture, in which the brain is composed of a fluctuating mosaic of neural activity as it adapts to changing sensory inputs. Therefore, dysfunction results not from 'punch out a module - lose a function', but by shifts in equilibria, which can be corrected, perhaps, by hitting a reset button. Even if this turns out to be true for a minority of syndromes, it will be well worth the effort.
\end{abstract}

\footnotetext{
${ }^{*}$ Corresponding author: Vilayanur Subramanian Ramachandran, Center for Brain and Cognition, 0109 Mandler Hall, UC San Diego, 9500 Gilman Drive, La Jolla, CA 92093-0109, United States. E-mail: vramachandran@ucsd.edu.
}

\section{The role of visual feedback}

Mirrors are inherently paradoxical - their properties have intrigued artists, puzzled philosophers, and been exploited by magicians for centuries. Only recently have they been found to even have thera- 
peutic benefits - and have been imported from the beauty parlor to clinic. - Ramachandran and RogersRamachandran (1998).

An early glimmer of an insight into this came decades ago when I examined a left arm amputee who complained of intermittent phantom limb pain. Like many patients he could voluntarily move his phantom - to answer a phone call, to shake hands, or to pet his puppy.

He could even reach out with his phantom hand to grab a coffee cup which had been placed an arms' length away on a table in from of him, volunteering that he could actually feel his fingers wrap around the handle. Then, a few minutes after he had let go of the cup, I placed it near him once again, asking him to reach out and grasp the cup. However, this time I pulled the cup away to see if his phantom arm would stretch out to anatomically impossible lengths to reach it. After all why should the physical limitations of flesh apply to a phantom?

To my surprise as I pulled the cup away, he winced in apparent pain and exclaimed "OUCH!" He explained he had already grabbed the cup with his fingers before I pulled it away, and that the pain was awful. He and I were both relieved that the pain lasted only seconds, but also surprised - that visual input could so profoundly influence phantom pain. Might not the converse be true: that you could relieve phantom pain using visual input?

Christian Dohle and his colleagues (Thieme et al., 2018) reviewed and analyzed over a decade of research on the role of mirrors in accelerating the recovery of function after stroke, and their clear conclusion is that the procedure works well, though there is considerable variation in the response. To optimize the effect one needs to explore which of the many variables enhances the effectiveness of the mirror. Morkisch et al. (in press) explore this in their new meta-analysis, to determine how different parameters of mirror therapy protocol influence the degree of recovery from hemiparesis after stroke. One major finding is that large mirrors work much better, possibly because this makes the illusion more compelling. This should be easy to implement in the clinic. Similarly, unilateral movements seem to work better than bilateral movements, which seems counter-intuitive, but would be wise to incorporate, pending further research. Lastly, they find that recovery in the absence of an object being manipulated was slightly more effective than when they manipulated an object.

Another way to optimize MT's efficacy would be to explore the characteristics of highly respon- sive "outlier" patients on whom the mirror works especially well. As we've noted before (Ramachandran \& Hirstein, 1995), there may be patients who may have especially large ipsilateral dormant corticospinal pathways which are activated by the mirror procedure. This can be determined by TMS on the motor cortex of each hemisphere, producing EMG activation of ipsilateral and contralateral limb muscles, prior to and after therapy.

\section{Plasticity in adult brains}

A long-standing dogma in neurology has been that connections in the brain are all laid down in fetal life or early infancy and there is no regeneration of neural pathways following damage to adult brains. It's no exaggeration to say that neurology was mainly an academic discipline concerned with "localizing the lesion" since very little could be done to regenerate lost tissue. This lack of plasticity in the adult brain was frustratingly obvious to patients, care-givers and physicians alike whether dealing with rare diseases such as the Capgras syndrome, or more common deficits like hemiparesis and neglect following stroke. Hence, the wide-spread pessimism was entirely justified. But what was NOT justified was that somewhere down the line, the fact that neural pathways do not regenerate was taken to mean that they CANNOT do so - and this is a logical fallacy.

This point is driven home extremely well by previous work done by Dohle and colleagues (as described below):

"Due to the existing bulk of evidence, further studies aiming to demonstrate the efficacy of MT as an adjunct therapy or compared to a placebo therapy are no longer necessary (Thieme et al., 2018). Rather, new studies should consider specific questions regarding the optimal application of MT after stroke. Ultimately, this should result in the definition of an optimum therapy protocol and an optimum target population for applying MT after stroke." (MT = mirror therapy)

It is especially noteworthy that out of 62 studies on mirror therapy with a total of 1982 participants, nearly all yielded statistically significant results to varying extent. Thieme et al. (2018) used rigorous criteria for inclusion in their analysis, vindicated by the Cochrane Central Register of Controlled Trials (CENTRAL). 


\section{Mirror visual feedback ("mirror box")}

During the decades spanning 1950 to around 2000 - a widely held superstition in neurology was that new connections cannot be formed in the adult brain. This started to be challenged by several independent lines of evidence converging on the same conclusion; namely the adult brain is plastic and the plasticity can be exploited clinically.

Morkisch et al.'s review (in press) is on stroke rehab, but the mirror box was first used for treating phantom pain. I will therefore describe those early studies to set the stage for this editorial, which is mainly on stroke.

There are multiple factors that contribute to phantom pain. One clue came from a review of the medical charts of these patients, and pertains to a phenomenon that we have dubbed "learned pain" and learned paralysis. By this, I mean the carry-over of preamputation pain into the phantom, especially when the amputation was performed months after a preexisting brachial plexus injury. Each attempt to move the intact paralyzed arm would have resulted in a visually observed inability to move (and proprioceptive signals that the arm isn't obeying the command). Therefore, a Hebbian link established between the very attempt to move the limb and ensuing pain results in a form of "learned pain" that is carried into the phantom. In this interpretation, the MVF acts by breaking the Hebbian link, i.e. "unlearning" the learned paralysis.

The second factor is the emergence of aberrant pathways and the resulting disharmony between different sensory inputs and motor commands that ordinarily harmonize to construct your dynamic body image, e.g. vision, proprioception, re-afference from motor commands, vestibular input. If so can the pain be reduced by restoring this congruence between these different systems, and removing the discrepancies? We initially thought of using VR technology to create the illusion that the patient was moving his missing or paralyzed hand in response to his motor commands but were discouraged by cost involved. We then realized that the same effect could be achieved by using a vertical parasagittal $\$ 5$ mirror propped on a table (Fig. 1). In this setup, the patient looks at the mirror reflection of the intact, normal (say, right) hand so that it is optically superposed on the FELT position of the phantom. If he then makes symmetrical movements (e.g. clenching, clapping, waving etc.) while looking at the reflection he gets the vivid impression the phantom was not only

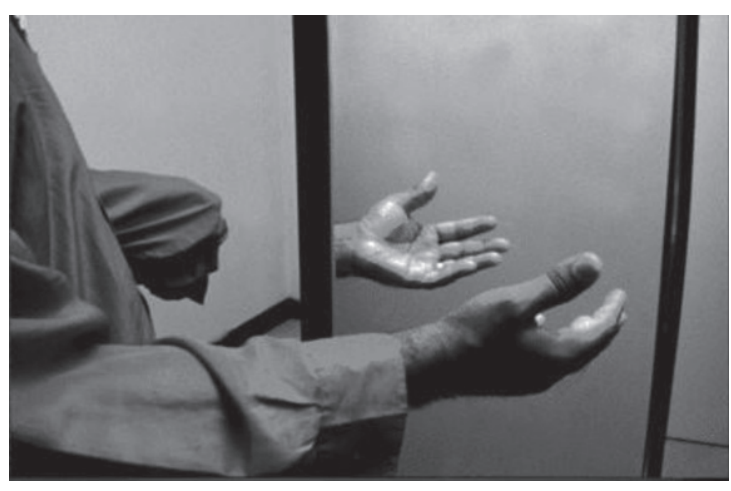

Fig. 1. Mirror box apparatus.

SEEN moving but FELT to move as well in precise synchrony with the commands, and in about half the cases, produces striking relief from pain. The control for this was just plexiglass instead of mirror. The vast majority of 62 clinical trials yielded statistically significant results. Of these, a majority were rigorously controlled trials (from the Cochrane database), which are the gold standard of rehab neurology.

\section{Cortical remapping, referred sensations and phantom pain}

Although Morkisch et al.'s meta-analysis of MVF is based on clinical work with patients, their optimism as well as ours was fueled by the pioneering animal experiments (Buonomano \& Merzenich, 1998; Devor \& Wall, 1981; Kaas, 1991; Gage, 2004) which showed that there is a great deal of latent plasticity in the adult primate brain. The most impressive of these was the demonstration that after complete surgical de-afferentation of an arm, the vacated territory gets 'invaded' by new sensory input originally destined for adjacent sensory areas representing other body parts (e.g. face). The same thing happens in human amputees, which we demonstrated using MEG mapping (Ramachandran, 1993). The vacated hand region is now activated by sensory input originally destined for the face area of cortex. Intriguingly, when you touch the patient's face he feels you touching his missing (phantom) hand with clear topography and well delineated "receptive fields" representing individual digits. Thus we showed that the Penfield map (Fig 2) in S1 can change with remarkable precision over $2 \mathrm{~cm}$ while preserving topography. Additionally if a drop of ice water dribbled down his cheek, the ice cold water could be felt dribbling down the phantom. When the stump pointed upward toward the ceiling, 


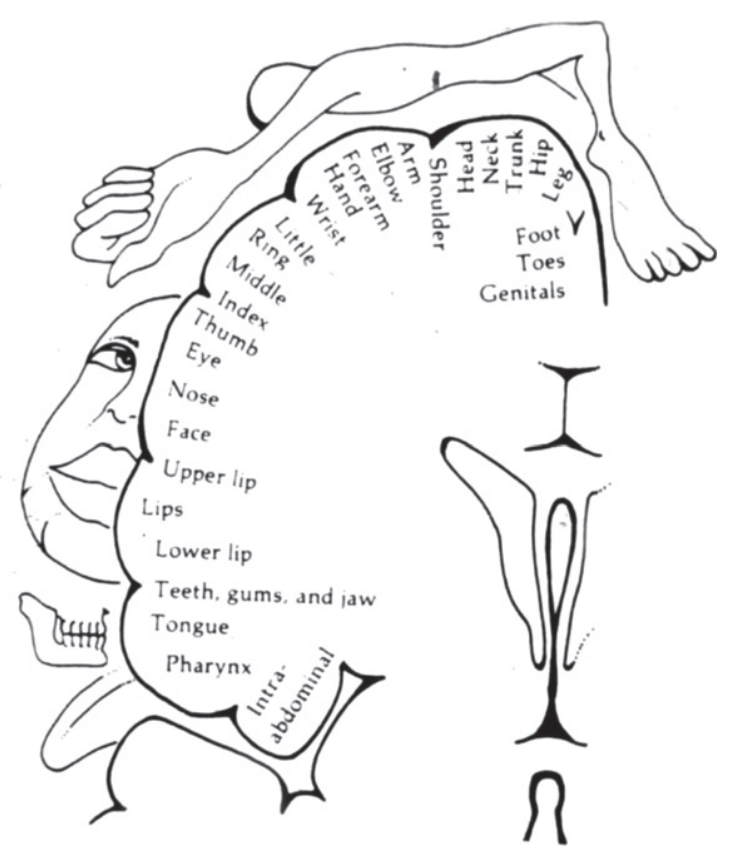

Fig. 2. Penfield map.

surprisingly, the water flowed uphill, defying the laws of gravity. Therefore connections can change in adult human brains and do so with high fidelity in a modality specific manner. If you put a hot teaspoon on the amputees face -its felt as a spot of heat on the phantom, whereas a piece of ice on the face generates precisely localized cold sensations - you never see "cross wiring", i.e. heat on the face being felt as cold on the hand. Hence, the reorganization is systematic - not higgledy-piggledy. The disposition of the three maps - touch, heat and cold, vary from patient to patient but are usually clustered the mandibular and lower maxilla with perfect congruence of the topography and reference fields of the three modalities (Fig. 3). The manner in which the congruence is achieved, perhaps by 'replaying' embryonic algorithms, is mysterious. And lastly, it's worth repeating that the cells in the original hand area receiving afferents from the face skin continue to signal "hand" the sensory quale depends entirely on output, and the cortex doesn't get relabeled "face" to accommodate the new input - surely an observation with quasi philosophical implications.

\section{Phantom pain}

As noted earlier, phantom pain probably has several origins (Sunderland, 1968; Mitchell, 1905). First,

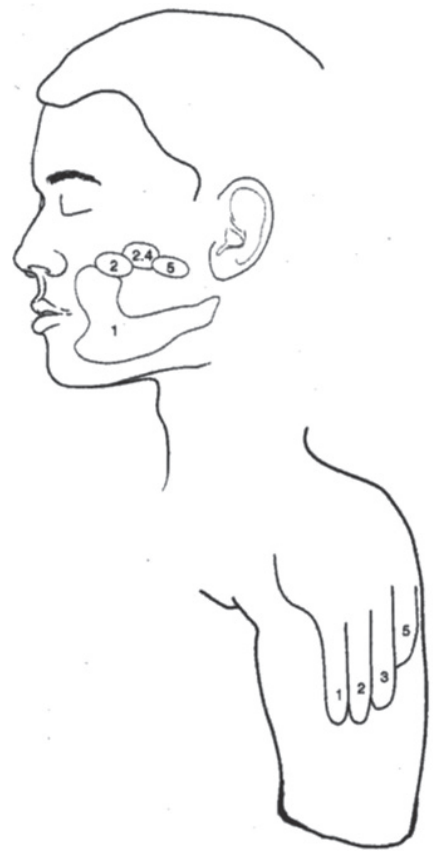

Fig. 3. Map of sensations referred from the face to the phantom hand.

just the clumps of scar-like tissue (i.e. neuromas) are undoubtedly part of the story; their activation could result in pain being felt in the phantom in some cases. But this in itself barely scratches the surface of the problem. Surgical removal of neuromas provides partial relief, and that too only in a minority of patients. But just as neuromas were overemphasized in the older literature, there is now a tendency to go to the other extreme, saying they play no role at all. The truth is half-way.

Second, though the remapping of hand to face is modality specific, some high threshold touch input might accidentally cross-activate low-threshold pain areas. Third, the remapping results in conflicting signals whose disharmony itself results in pain (Harris 1999) (e.g. reafference signals sent to somatosensory cortex feedback from S1, S2, M and V1, and lack of proprioceptive confirmation) Fourth, the alien input is functionally effective in activating new territory, but the synapses may not be quite normal, and their signals might be interpreted as junk - or phantom pain.

The connection between cortical reorganization and pain is a complex one, and is being tackled by Herta Flor and her colleagues (Flor et al., 1995). They found a clear-cut correlation between extent of remapping and the extent of referral of neutral sensations. This remapping in turn provides greater 
opportunity for aberrant connections and the misfiring that contributes to pain. However in some patients, pain dominates the clinical picture and is so intense that it can actually mask referred neutral sensations (Ramachandran \& Hirstein, 1995). The exact manner in which neutral and painful sensations interact with proprioception (now missing since the arm is gone) as well as re-afference from motor commands is poorly understood. Such understanding is essential if we are to reduce pain and paralysis and improve the lifestyles of millions of patients across the globe. Mirrors are not nearly as effective as (say) antibiotics for infections, but are arguably more effective than (say) SSRIs for depression, or invasive surgery or the oxycontin that is currently prescribed indiscriminately for chronic pain (at least you can't get addicted to mirrors!).

\section{Hemiparesis and post-stroke pain}

Given the success of MVF in treating phantomlimb pain (PLP); it occurred to us that perhaps other types of paralysis may have a functional component, as in many cases of stroke. Following a thrombosis or hemorrhage there is immediate tissue death that cannot be reversed. However, a large component of the paralysis might be the temporary inhibition or compression of normal motor tracts and cells, caused by the adjacent inflamed, swollen tissue. This is usually reversible, but in an unfortunate subset, say $10 \%$, the paralysis persists even though the swelling has long subsided - an example of the "learned paralysis" i.e., the link between the command to move the limb and it's the inability to budge leads to a form of pseudo-paralysis.

If so, can we accelerate recovery using MVF by making it look like his arm is obeying his command with impunity - thereby restoring the loop? In a small blind trial of 9 patients my colleagues and I found this to be the case, and published this work in the LANCET (Altschuler et al., 1999) - informing healthcare professionals who were unaware of the fact that mirrors show some promise toward recovery from post-stroke hemiparesis. Subsequently it was found that even sensory loss in the skin surface showed substantial recovery.

The sensory recovery, though surprising in the context of medical school Neuroanatomy 101, makes perfect sense in our scheme which blurs the distinction between motor and sensory, as exemplified in the discovery of mirror neurons2005 (Rizzolatti, 2005).
More recently neglect as well as post-stroke pain (Dejerine-Roussy) have been shown to respond well to MVF (Ramachandran et al., 1999; Pandian et al., 2014), as have rehab after hand surgery and cerebral palsy (Feltham et al., 2010).

\section{Complex regional pain syndrome (CRPS)}

Mirrors reducing pain in a phantom is surprising enough, but even more remarkable is the wealth of accumulating evidence that real pain in a real (intact) limb can also benefit from using mirrors, as in complex regional pain syndrome (CRPS) (even more effectively than its efficacy with phantoms).

CRPS is an enigmatic disorder whose cause is unknown - though it results from pathological overreaction as well as prolonged persistence, sometimes for years or decades, of pain and inflammation long after the inciting injury has healed. The normal sequence after, say, metacarpal bone hairline fracture, is a reflex immobilization and severe pain of the finger - an adaptive reflex to keep the finger out of harm's way, along with edema, color and temperature change. In neurotypicals the bone heals and in a couple of weeks the swelling subsides (reverses!), the temperature drops to 98.5 degrees, and the inflammation goes away. But in a substantial minority $(5 \%)$ long after the bone fracture has healed, the swelling and inflammation become worse, the temperature goes up, and the pain becomes unbearable. The origin of RSD pain (Reflex Sympathetic Dystrophy, also known as CRPS) is obscure but I suggested in 1995 that a major contributing factor may be a form of learned pain which can perhaps be unlearned by using mirrors. Sure enough, it was soon afterwards discovered by a group of pioneering researchers (McCabe et al., 2003) that MVF can produce relief from the intense pain of CRPS, which remains effective at least 6 months after therapy. What is especially striking is that the swelling, pain, skin color and temperature were all observed to change in real time as the patient was being watched - the most striking illustration of mind-body interactions that I know of. I raised my skeptical eyebrows until I noticed one of the authors was Patrick Wall, arguably the world's pre-eminent expert on pain and placebos.

Other disorders treated with MVF include hand rehab recovery from surgery, pioneered by Eric Altschuler, instability of gait post-stroke, and cerebral palsy. These last ones have not yet been rigorously tested under controlled conditions, but the results 
look promising, so one should remain cautiously optimistic.

\section{Vestibular input modulates pain in amputees and dejerine-roussy central pain syndrome}

Two additional observations are also useful for demonstrating the plasticity of the adult CNS and its impact, both in the clinic and in the laboratory. While the maps are largely stable for days or weeks, we were surprised to note that they can be made to shift by as much as a $2 \mathrm{~cm}$ if the patient simply pronates or supinates the phantom - but this effect is seen only for touch, never for heat and cold perception. Secondly, given that there is a strong vestibular projection to the inferior parietal lobule (e.g. vestibular cortex, insula, etc.) contributing to the construction of body image - we predicted and found that stimulating the vestibular system using caloric irrigation and GVS (galvanic vestibular stimulation) substantially altered the overall shape, size and location of receptive fields (reference fields) for several minutes; it would appear that even though the maps are mostly stable, we should regard them not as fixed anatomical entities, but as dynamic fluctuating mosaics of activity. Perhaps the concept of a receptive field has outlived its utility, and should henceforth be called 'deceptive fields'. One recalls the ingenious experiments launched by the late Tim Pons showing in monkeys that cooling the S1 and S2 cortices produces a 6- to 8-fold increase in the size of thalamic receptive fields, which are purportedly constructed at an earlier stage of processing.

The striking effects of GVS-induced vestibular activation on body image are well known. They led me and my colleague Paul McGeoch to predict that such procedures might be of use in treating thalamic pain or Dejerine-Roussy syndrome (Ramachandran, McGeoch and Williams, 2007; Ferré, Vagnoni, and Haggard, 2013), which is largely incurable and characterized by unremitting intense pain originating in the thalamus, usually post-stroke. We have now tested this conjecture on seven patients with chronic, relentless pain lasting several months or years, on whom none of half a dozen conventional remedies such as narcotics or TENS units had any significant effect (compared to placebos). Yet a single 'dose' of caloric irrigation or GVS produced striking recovery in half the patients - an effect now confirmed by other groups (Ferrè et al., 2015; Spitoni et al., 2016). Unfortunately, the effects usually lasted only for a few hours or (in two cases) for weeks, after which the pain returned. Whether it turns out to be a 'cure' or not, it should help us achieve a deeper understanding of pain mechanisms - which remain the terra incognita of brain research.

\section{The dynamic brain: receptive fields or deceptive fields}

Dohle's work, along with those of physiologists and physicians, has opened up several new avenues of treatment that complement rather than replace pharmacological approaches. But their implications go far beyond their clinical utility and radically alter the way we think of normal brain function (Ramachandran, 2005). Until two decades ago, it was widely believed that the brain is composed of several highly specialized autonomous modules arranged in a serial hierarchy; each module computes and makes explicit some aspect of the information and sends the result to the next module higher up, till you reach the highest levels of abstraction, culminating in cognitive functions like language and thought. The flow of information was unidirectional, the modules were hardwired and autonomous. We have dubbed this the serial hierarchical bucket brigade model of brain function, which should now be replaced by a more dynamic picture - one that conforms to the way evolution really works - simultaneously opportunistic and conservative. In this view, the modules are not hardwired, but in fact in a state of dynamic equilibrium, with the environment and with other modules, constantly changing their connections to meet new environmental challenges.

Furthermore the flow of information is not always centripetal - at each stage of processing, the result is sent back to bias earlier stages, which would tremendously reduce the "problem space" as in a twenty questions game. Without such iterations, the problem of perception is under-constrained and would lead to a combinatorial explosion. Add to the equation the fact that the modules along a hierarchy also interact sideways with other parallel modules (e.g. mirrors curing somatic pain), with the skin and bones (as in CRPS) and even with the brains of other people (via mirror neurons) and you have a picture of brain function that is utterly different from computers. Real brains deploy multiple crude shortcuts because they are easier to evolve than a single sophisticated algorithm (since evolution has no foresight, it strives for adequacy not optimality). Additionally, it buys you 
noise-tolerance - like two drunks who cannot cross the road individually, but by leaning on each other's shoulders, reach their destination.

Every medical student is taught different specialized modules are hardwired for specific functions which once destroyed, cannot be replaced. However, it should be clear from the last two decades of research that the "punch out a module - lose a function" model that used to be part of folk-neurology - though still useful as a first-approximation - must be replaced by a very different picture; namely, one in which brain modules are in a constant state of equilibrium with each other, and disease often result from shifts in equilibrium than from permanent loss. If so, one of our research strategies should be to find the right 'switch' to restore equilibrium - whether it be a mirror, or cold water in the ear. Given that 1 out 6 people worldwide will suffer have a stroke, even if only a minority of syndromes respond to such an approach, it would have been well with the effort.

\section{Acknowledgments}

We thank Dr. Eric Altschuler who has been our longstanding collaborator in many of our studies involving the utility of mirrors.

\section{References}

Altschuler, E.L., Wisdom, S.B., Stone, L., Foster, C., Galasko, D., Llewellyn, D.M.E., \& Ramachandran, V.S. (1999). Rehabilitation of hemiparesis after stroke with a mirror. The Lancet, 353(9169), 2035-2036.

Bach-y-Rita, Paul. (1967) "Sensory plasticity: Applications to a vision substitution system." Acta Neurologica Scandinavica, 43.4, 417-426.

Buonomano, D.V., \& Merzenich, M.M. (1998). Cortical plasticity: From synapses to maps. Annual review of neuroscience, 21(1), 149-186.

Devor, M.A.R.S.H.A.L.L., \& Wall, P.D. (1981). Plasticity in the spinal cord sensory map following peripheral nerve injury in rats. Journal of Neuroscience, 1(7), 679-684.

Feltham, M.G., Ledebt, A., Deconinck, F.J., \& Savelsbergh, G.J. (2010). Mirror visual feedback induces lower neuromuscular activity in children with spastic hemiparetic cerebral palsy. Research in developmental disabilities, 31(6), 1525-1535.

Ferrè, E.R., Vagnoni, E., \& Haggard, P. (2013). Vestibular contributions to bodily awareness. Neuropsychologia, 51(8), 14451452. https://sciencedirect.com/science/article/pii/S0028393 213001218

Ferrè, E.R., Haggard, P., Bottini, G., \& Iannetti, G.D. (2015). Caloric vestibular stimulation modulates nociceptive evoked potentials. Experimental brain research, 233(12), 3393-3401.
Flor, H., Elbert, T., Knecht, S., Wienbruch, C., Pantev, C., Birbaumers, N.,... \& Taub, E. (1995). Phantom-limb pain as a perceptual correlate of cortical reorganization following arm amputation. Nature, 375(6531), 482.

Gage, F.H. (2004). Structural plasticity of the adult brain. Dialogues in clinical neuroscience, 6(2), 135.

Harris, A.J. (1999). Cortical origin of pathological pain. Lancet, 354(9188), 1464-1466. https://www.ncbi.nlm.nih.gov/pub $\operatorname{med} / 10543687$

Kaas, J.H. (1991). Plasticity of sensory and motor maps in adult mammals. Annual review of neuroscience, 14(1), 137-167.

McCabe, C.S., Haigh, R.C., Ring, E.F.J., Halligan, P.W., Wall, P.D., \& Blake, D.R. (2003). A controlled pilot study of the utility of mirror visual feedback in the treatment of complex regional pain syndrome (type 1). Rheumatology, 42(1), 97-101.

Mitchell, S.W. (1905). The Autobiography of a Quack and the Case of George Dedlow. Century Company.

Morkisch, N., Thieme, H., \& Dohle, C. (2019). How to perform mirror therapy after a stroke? Evidence from a meta-analysis. Restorative Neurology and Neuroscience, 37(5), 421-435.

Ramachandran, V.S. (1993). Behavioral and magnetoencephalographic correlates of plasticity in the adult human brain. Proceedings of the National Academy of Sciences of the United States of America, 90(22), 10413.

Ramachandran, V.S., \& Hirstein, W. (1998). The perception of phantom limbs. The DO Hebb lecture. Brain: A journal of neurology, 121(9), 1603-1630.

Ramachandran, V.S. (2005). Plasticity and functional recovery in neurology: Oliver-Sharpey Lecture, Royal College of Physicians. Clinical Medicine, 5(4), 368-373.

Ramachandran, V.S., McGeoch, P.D., \& Williams, L. (2007). Can vestibular caloric stimulation be used to treat Dejerine-Roussy Syndrome?. Medical hypotheses, 69(3), 486-488.

Ramachandran, V.S., \& Altschuler, E.L. (2009). The use of visual feedback, in particular mirror visual feedback, in restoring brain function. Brain, 132(7), 1693-1710.

Rizzolatti, G. (2005). The mirror neuron system and its function in humans. Anatomy and embryology, 210(5-6), 419-421.

Sathian, K., Greenspan, A.I., \& Wolf, S.L. (2000). Doing it with mirrors: A case study of a novel approach to neurorehabilitation. Neurorehabilitation and neural repair, 14(1), 73-76.

Spitoni, G.F., Pireddu, G., Galati, G., Sulpizio, V., Paolucci, S., \& Pizzamiglio, L. (2016). Caloric vestibular stimulation reduces pain and somatoparaphrenia in a severe chronic central poststroke pain patient: A case study. PloS one, 11(3), e0151213.

Sunderland, S. (1968). Nerves and nerve in juries. E \& S Livingstone Ltd, Edinburgh.

Thieme, H., Morkisch, N., Mehrholz, J., Pohl, M., Behrens, J., Borgetto, B., \& Dohle, C. (2018). Mirror therapy for improving motor function after stroke. Cochrane Database of Systematic Reviews, (7). 